Pensamiento Crítico Vol. 21, No 2, pp. 145 - 154

\title{
La gestión de la innovación tecnológica en las empresas
}

\author{
Management of technological innovation in companies
}

[Recepción: Setiembre de 2016 / Conformidad: Octubre de 2016]

Jazmín Tavera Colonna ${ }^{1}$

\section{RESUMEN}

La innovación tecnológica enfrenta en nuestro país más que un problema de recursos; es un problema de gestión, que en las empresas se traduce en el desarrollo de las capacidades de inversión, producción y vinculación. El desarrollo de estas capacidades requiere de una estrategia de innovación tecnológica. Para los sectores metalmecánico y tecnologías de la información mexicanos se confirma la existencia de una relación directa entre la gestión tecnológica y la capacidad tecnológica. Las universidades y facultades, como la de economía, tienen un rol hoy en día desatendido en estas estrategias.

Palabras clave: Gestión, innovación, capacidades empresariales, estrategia.

\section{ABSTRACT}

Technological innovation face in our country more than a resource problem, is a management problem, which results in companies developing capabilities of investment, production and bonding. The development of these capabilities

1 Economista, magíster en Economía y maestra en Ciencias. Docente de la Facultad de Ciencias Económicas de la UNMSM. Directora ejecutiva del Grupo de Estudios y Capacitación. 


\section{Pensamiento Crítico Vol. ᄅl, № 2}

requires a strategy of technological innovation. For mexican metalworking and information technology sectors confirms the existence of a direct relationship between technology management and technological capabilities. Universities and colleges, such as Economy, have a role today unattended in these strategies.

Keywords: Management, innovation, business skills, strategy.

\section{Introducción}

De acuerdo al índice global de competitividad del World Economic Forum (WEF), la tecnología es un parámetro de competitividad, en tanto es la base sobre la que se sostienen los 12 pilares de la competitividad, comprometiendo directamente al pilar 9 Formación Tecnológica y al pilar 12 Innovación.

Tal como se puede identificar en el Esquema 1, el pilar que mostró mayor debilidad para Perú es innovación, lo que definitivamente no nos permite observar una mejor posición en el ranking, manteniéndonos en la etapa de desarrollo 2. Es, además, significativamente preocupante que los dos indicadores que siguen en bajo score sean institucionalidad y formación tecnológica.

\section{ESQUEMA 1}

Perú: Índice de Competitividad Global

Global Competitiveness Index

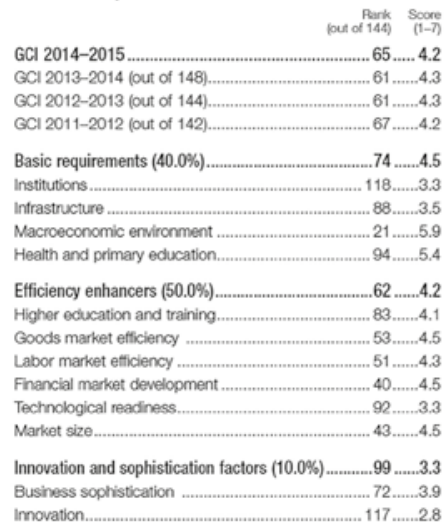

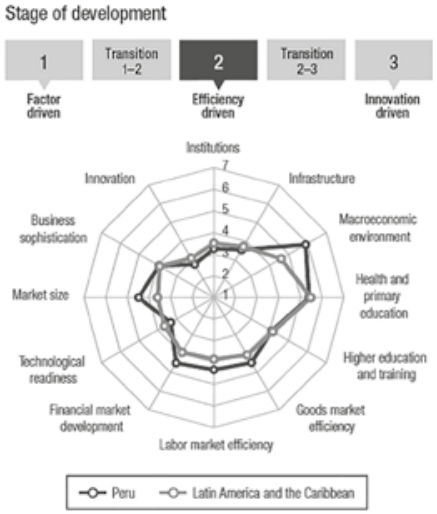

Fuente: WEF Global Competitiveness Index 2015. 


\section{Jazmín Tavera Colonna}

Y si bien América Latina invierte poco en I \& D, la inversión que realiza Perú es de las más bajas de la región, superando solo a los países centroamericanos (ver cuadro 1). Si estos resultados los relacionamos con lo que recibimos en dólares por el uso de nuestras patentes en el exterior, se establece una relación directa. Así tenemos que Perú recibe unos 5,3 millones de dólares; Bolivia, 8,5 millones; Colombia, 89,7 millones; Chile, 75,4 millones; México, 95,6 millones; Argentina, 161,8 millones y Brasil, 510,7 millones de dólares.

Mas si añadimos el porcentaje de exportaciones de alta tecnología respecto a exportaciones totales, la relación con inversión en I \& D ya no es directa. Ni El Salvador, con un PBI y un porcentaje de inversión en I \& D menor al peruano, exporta una proporción de exportaciones de alta tecnología tan pequeña como la peruana. Por lo tanto, el problema del desarrollo tecnológico peruano no solo está en el bajo porcentaje de inversión en I\&D. El sistema de innovación peruano ha de tener serios problemas de gestión (Tavera, 2014).

En Perú, los sectores más innovadores con actividades de innovación por encima del $75 \%$ son: alimentos $(97,4 \%)$, farmacéuticos $(82,1 \%)$, bebidas $(79,4 \%)$, cuero y calzado $(75,7 \%)$ y metalmecánica $(75,5 \%)$. Por debajo de $50 \%$ están prendas de vestir, muebles y otros equipos de transporte, y menos de $40 \%$ sustancias y productos químicos. El sistema de innovación parece no funcionar en el Perú aunque la innovación existe, limitada y concentrada en ciertos sectores

CUADRO 1

Proporción del PBI destinada a I \& D y proporción de las exportaciones de alta tecnología respecto a las exportaciones totales, 2009-2013

\begin{tabular}{|l|c|c|}
\hline \multicolumn{1}{|c|}{ Países } & \%I\&D / PBI & \%X AT/XTotal \\
\hline Alemania & 2,89 & 16 \\
\hline España & 1,36 & 7 \\
\hline Finlandia & 3,8 & 9 \\
\hline EE.UU. & 2,76 & 18 \\
\hline China & 1,98 & 26 \\
\hline Singapur & 2,23 & 45 \\
\hline Argentina & 0,65 & 8 \\
\hline
\end{tabular}




\section{Pensamiento Crítico Vol. ᄅl, $N^{\circ} 2$}

\begin{tabular}{|l|c|c|}
\hline Brasil & 1,21 & 10 \\
\hline Bolivia & 0,16 & 9 \\
\hline Chile & 0,37 & 5 \\
\hline Colombia & 0,17 & 5 \\
\hline El Salvador & 0,08 & 5 \\
\hline México & 0,43 & 16 \\
\hline Perú & 0,15 & 3 \\
\hline Uruguay & 0,43 & 6 \\
\hline
\end{tabular}

Fuente: Tavera, (2014)

\section{GRÁFICO1}

Eficiencia del Synacit por nivel de aceptación de usuarios

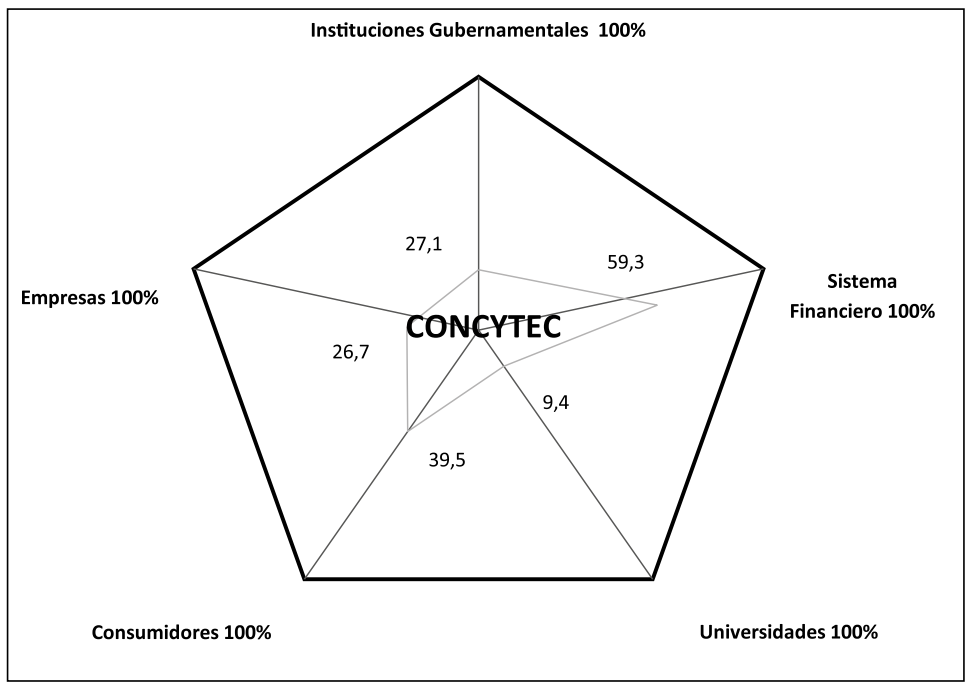

Fuente: Tavera (2015)

A partir de los resultados obtenidos de la encuesta nacional de innovación en la industria manufacturera 2012, encontramos que el sector mejor integrado es el financiero, con 59,3\% de aceptación, le sigue la relación con los consumidores, con una aceptación de usuarios de $39,5 \%$, donde la dinámica corresponde básicamente al consumidor de mercado externo. 


\section{Jazmín Tavera Colonna}

Las instituciones gubernamentales tienen un nivel de aceptación muy bajo (dado por el área delimitada internamente en el pentágono), 27,1\%. Además de los altos costos, los tiempos excesivos y la complejidad administrativa de la protección formal, con la que las empresas califican el mal accionar del Estado, este brinda programas de capacitación. Entre estos, los de mayor aceptación son los referidos al entrenamiento de personal, la promoción de exportaciones y los programas de mejora de la calidad y la obtención de conformidad con norma certificación, programas con un nivel de acceso de $88,5 \%$ pero cuya aceptación no supera el $24,3 \%$. En lo que respecta al apoyo que brindan los CITE - Senati, así como los institutos de investigación públicos, las empresas los reconocen entre un $25,2 \%$ y $2,2 \%$ respectivamente (Produce, 2013).

Freeman (2002), al analizar a países latinoamericanos como Brasil, Argentina y Uruguay, encuentra que las relaciones que establecen las empresas con otras empresas, así como los vínculos con el sistema de ciencia y tecnología, son decisivos. En nuestro caso, estas interrelaciones no sobrepasan un nivel de aceptación de $26,7 \%$. Y es que el sistema no se basa en un desarrollo sectorial articulador. Países como Brasil, India, Argentina han encontrado en la industria automotriz, electrónica y de bienes de capital sus programas articuladores, sectores cuya dinámica se sostiene en el mercado interno y otros de similar o menor desarrollo en el exterior. Nuestro marco institucional, el Plan Nacional Estratégico de Ciencia, Tecnología e Innovación para la Competitividad y el Desarrollo Humano 2006-2021 se concentra en áreas prioritarias, sin identificar ejes articuladores, a pesar de que su objetivo es asegurar la articulación y concertación entre los actores del Sinacyt para "atender las demandas tecnológicas en áreas estratégicas prioritarias, con la finalidad de elevar el valor agregado y la competitividad, mejorar la calidad de vida de la población y contribuir con el manejo responsable del medio ambiente".

Finalmente, la universidad, agente con rol decisivo en el desarrollo de las oleadas tecnológicas en Occidente y Oriente, observa el menor nivel de aceptación, 9,4\%. La universidad pública tiene un rol crítico en la oferta de recurso humano de calidad $e$ idóneo, ya que, por la oferta de carreras que tiene, es el segundo factor limitante para la innovación. Su rol como mecanismo de movilidad social requiere también de una reingeniería estructural administrativa, reestructuración curricular y capacitación integral de sus docentes para una profesionalización adecuada de los estudiantes. Hay, por tanto, a nivel de todo el sistema de innovación, limitaciones de infraestructura, presupuesto, 


\section{Pensamiento Crítico Vol. ᄅl, № 2}

pero ante todo, de gestión de la tecnología, de la innovación y, por supuesto, del conocimiento, que es la base de la innovación y la tecnología.

\section{La gestión de la innovación tecnológica en las empresas.}

De acuerdo a Mendoza y Valenzuela (2013), citando a Sanjaya Lall, se parte de que el desarrollo tecnológico y la innovación no son estadios momentáneos, sino procesos que requieren del aprendizaje para que, por medio del fortalecimiento o incremento de recursos, o la acumulación de capacidades, se refuercen tres funciones: inversión, producción y vinculación o soporte. Cada una de estas funciones tiene un núcleo básico que debe ser internalizado por la empresa para posteriormente crecer con ella, a medida que sus actividades se hacen más complejas.

De acuerdo a Ortega (2005), la inversión es una capacidad que permite identificar, preparar y obtener las tecnologías indispensables para el diseño, la construcción y el equipamiento de una nueva planta o su ampliación, además de las capacidades de reclutamiento de personal. Esta función permite definir la escala de producción, la tecnología a emplear, los costos de capital del proyecto y el nivel de entendimiento que la empresa ha ganado sobre las tecnologías básicas empleadas.

La función de producción abarca actividades básicas como control de calidad, operación y mantenimiento, así como las más avanzadas: adaptaciones, alargamiento de la vida útil de los equipos, investigación, diseño e innovación. Esta función o capacidad, como la llama Ortega (2005), permite comprender la operación de la tecnología, así como identificar los esfuerzos internos de la empresa para absorber las tecnologías adquiridas o imitadas. Tanto la función de inversión como la de producción, se consideran cono funciones primarias, siendo estas las que generan el cambio técnico y administran su implementación.

La función de vinculación o soporte es una capacidad que permite recibir y transmitir información, experiencia y tecnología de los proveedores, consultores, consumidores, sistema financiero y universidades a la empresa. Si la función de inversión afecta la eficacia, la de vinculación está directamente relacionada con la eficiencia productiva de la empresa y su capacidad productiva (Ortega, 2005). 


\section{Jazmín Tavera Colonna}

El aprendizaje es un proceso dinámico de adquisición y construcción de estas funciones o capacidades tecnológicas en forma gradual y acumulativa, depende del flujo de conocimiento dentro de la empresa, entre empresas (sector) y con su entorno. En el caso de países en desarrollo que no elaboran estas tecnologías, es imprescindible que estas capacidades sean construidas para poder internalizar las tecnologías adquiridas a sus procesos.

Las empresas pueden desarrollar trayectorias dinámicas de acumulación de capacidades tecnológicas a partir de una deliberada estrategia tecnológica (Ortega, 2005). Con la gestión tecnológica, la empresa podrá obtener, acumular y procesar la información y convertirla en conocimiento.

Mendoza y Valenzuela (2014) definen la gestión de la tecnología como "la organización y dirección de recursos humanos y económicos para aumentar la creación de nuevos conocimientos; es también la generación de ideas técnicas que permitan obtener nuevos productos, procesos y servicios o mejorar las ya existentes, el desarrollo de dichas ideas en prototipos de trabajo y su transferencia a las fases de fabricación, distribución y uso". Esta gestión tiene tres niveles claramente diferenciados: dirección estratégica, transferencia tecnológica e innovación tecnológica.

ESQUEMA 2

El proceso de aprendizaje
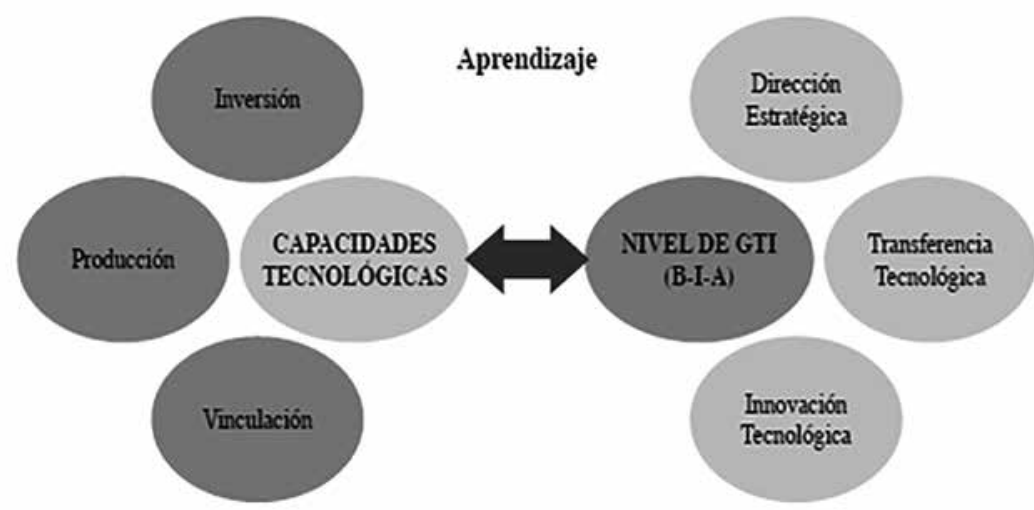

Fuente: Mendoza y Valenzuela (2014). 


\section{Pensamiento Crítico Vol. ᄅl, № 2}

Lall define las capacidades tecnológicas por medio de matrices; Bell y Pavitt implementan una nueva taxonomía para esas capacidades, lo que se resume en el siguiente esquema.

\section{ESQUEMA 3}

Matriz de capacidades tecnológicas de Lall - Bell y Pavitt

\begin{tabular}{|l|l|l|}
\hline \multicolumn{1}{|c|}{$\begin{array}{c}\text { Funciones } \\
\text { técnicas }\end{array}$} & \multicolumn{1}{c|}{ Variables } & \multicolumn{1}{c|}{ Profundidad } \\
\hline De inversión & $\begin{array}{l}\text { 1. Toma de decisiones y control } \\
\text { 2. Preparación y ejecución de proyectos }\end{array}$ & Innovativas básicas \\
\hline De producción & $\begin{array}{l}\text { 1. Centradas en el proceso } \\
\text { 2. Centradas en el producto }\end{array}$ & Innovativas intermedias \\
\hline De soporte & $\begin{array}{l}\text { 1. Vinculación interna } \\
\text { 2. Vinculación externa } \\
\text { 3. Desarrollo de equipo }\end{array}$ & Innovativas avanzadas \\
\hline
\end{tabular}

Fuente: Mendoza y Valenzuela (2014).

La gestión de la tecnología en los sectores metalmecánico y tecnología de la información

Según los esquemas 2 y 3, Mendoza y Valenzuela (2014) elaboran un cuestionario que se aplica a 672 empresas cuya principal actividad es la metalmecánica y tecnologías de la información, en las que se observa, de manera clara, los procesos de aprendizaje e innovación tecnológica; son micro, pequeñas y/o medianas empresas y se encuentran dentro de los cinco municipios seleccionados del estado de Sonora, noroeste de México.

De este total de la muestra, el 86,5\% asegura realizar innovaciones. El 55\% considera que sus innovaciones están sobre el promedio y $31 \%$ considera que está en la frontera tecnológica. Solo $18 \%$ considera que está por debajo del promedio. Estas disparidades se explican, de acuerdo a los autores, porque la obsolescencia llega más retrasada en los equipos de una empresa que en los personales: "el plazo de obsolescencia es mucho mayor en una computadora personal (PC) que en una máquina de control numérico computarizado (CNC)" (Mendoza y Valenzuela, 2014). 


\section{Jazmín Tavera Colonna}

El análisis econométrico que aplican permite identificar la probabilidad de relaciones estructurales entre las diversas variables. El modelo de regresión simple arroja una relación directa entre la gestión tecnológica y la capacidad tecnológica, tal como se aprecia en los resultados que presentan:

$$
\begin{aligned}
& \mathrm{CT}=0.103+0.751 \mathrm{GT}+\mathrm{u}_{\mathrm{i}} \\
& (0.019)(0.043) \\
& \mathrm{t} 5.29817 .411 \\
& \mathrm{R}^{2}=0.727 \\
& \mathrm{~F}=303.143
\end{aligned}
$$

De acuerdo a estos resultados, por cada unidad que aumenta el índice de gestión tecnológica, se dará un aumento en 0,751 puntos en el índice de capacidades tecnológicas.

\section{Conclusiones}

1. La tecnología suele ser tratada en economía como un parámetro, una caja negra que consideramos es materia de las ciencias ingenieriles. Sin embargo, dada la alta dependencia de las dinámicas de crecimiento de la variable tecnológica, es necesario que actuemos sobre ella.

2. La gestión de la innovación tecnológica es un campo en desarrollo que da las herramientas para lograr no solo procesos eficaces, sino también eficientes, y, por ende, permite desarrollar la senda de crecimiento prevista. Para ello, es necesaria una adecuada dinámica de aprendizaje tecnológico en las empresas y en las universidades, el cual es aún muy exiguo en nuestro país.

3. El rol de las universidades y sus facultades está en coadyuvar a que estas funciones empresariales se puedan desarrollar adecuadamente en un contexto innovativo, replanteándose su dinámica y estructura, haciendo una reingeniería de su institucionalidad, sus profesores y su alumnado. 


\section{Pensamiento Crítico Vol. 己l, № 2}

\section{Referencias bibliográficas}

Freeman, C. (2002). Continental, national and sub-national innovation systems complementarity and economic growth. Research Policy, 31 (2)

Freeman, C. (1987). Technology Policy and economic performance. Lessons from Japan. Londres: Ed. Pinter.

Lundvall, B. (1985). Product Innovation and user producer interaction. Industrial Development Research Series, 31.

Mendoza León, J. y Valenzuela Valenzuela, A. (2014). Aprendizaje, innovación y gestión tecnológica en la pequeña empresa. Un estudio de las industrias metalmecánica y de tecnologías de información en Sonora. Contaduría y Administración, 59 (4)

Ministerio de la Producción (2013) Encuesta Nacional de la Industria Manufacturera 2012

World Economic Forum (2016) WEF Global Competitiveness Index 2015.

Ortega, R. (2005) Aprendizaje y acumulación de capacidades tecnológicas en un grupo del sector siderúrgico. Innovar, Revista de Ciencias Administrativas y Sociales. Universidad Nacional de Colombia

Tavera, J. (2015). ¿Es eficiente el sistema de gestión tecnológico peruano? Pensamiento Crítico(20), IIECO, FCE, UNMSM

Tavera, J. (2014). El estado de la innovación tecnológica en el Perú. Revista de Economía San Marcos,1 (1), FCE, UNMSM. 